\title{
Measurement system design for civil infrastructure using expected utility
}

\author{
Romain Pasquier ${ }^{\mathrm{a}, *}$, James-A. Goulet ${ }^{\mathrm{b}}$, Ian F. C. Smith ${ }^{\mathrm{a}}$ \\ ${ }^{a}$ Applied Computing and Mechanics Laboratory (IMAC), School of Architecture, Civil and Environmental Engineering \\ (ENAC), Swiss Federal Institute of Technology (EPFL), CH-1015 Lausanne, Switzerland \\ ${ }^{b}$ Department of Civil and Geologic and Mining Engineering, Polytechnique Montréal, Montréal, H3T 1J4, Canada
}

\begin{abstract}
For system identification, most sensor-placement strategies are based on the minimization of the modelparameter uncertainty. However, reducing the uncertainty in remaining-life prognosis of structures is often more relevant. This paper proposes an optimization strategy using utility theory and probabilistic behavior prognoses based on model falsification to support decisions related to monitoring interventions. This approach, illustrated by the full-scale case study of a bridge, allows quantification of the expected utility of measurement systems while also indicating the profitability of monitoring actions. In addition, this approach is able to determine when the expected performance of monitoring configurations is reduced due to over-instrumentation. The use of model falsification for system identification allows for explicit inclusion of engineering heuristics in this knowledge intensive task while also offering robustness to effects of systematic modeling errors that are associated with idealization of complex civil structures.
\end{abstract}

Keywords: Sensor placement, model falsification, utility function, optimization, fatigue assessment, actualized repair cost.

\section{1. Introduction}

With the aging of infrastructure and limited investments allocated to structural management, an increas3 ing number of existing structures are measured. Efficient strategies are required to evaluate the performance 4 of measurement systems prior to site measurements. Such information helps owners prioritize monitoring 5 actions. Several researchers have observed a tendency to over-instrument structures and have noted the need 6 for fewer and more judiciously placed sensors $[3,7]$. In order to avoid over-instrumentation and improve 7 performance of data interpretation, measurement-system-design strategies have the potential to support

${ }^{*}$ Corresponding author. Address: EPFL ENAC IIC IMAC, Station 18, CH-1015 Lausanne, Switzerland. Email address: rpasquie@gmail.com (Romain Pasquier) 
the selection of good combinations of sensor types, locations and measurement scenarios, such as load test configurations.

Since the optimal design of a measurement system is dependent on what is done with the data, the characteristics of data-interpretation strategies need to be included in the development of sensor-placement algorithms. Several contributions in the field of system identification have based the search for optimal sensor placement on information theory for the purpose of improving parameter estimation. Some authors have maximized the determinant of the Fisher information matrix to obtain the optimal sensor configuration $[10,29,34]$. Others studied entropy-based approaches [12, 16, 17, 30]. Information entropy and the Fisher information matrix quantify the information associated with model parameters. Although entropy-based approaches have shown to be powerful for optimizing measurement systems to improve predictions, few studies have included systematic modeling uncertainties caused by simplifications and omissions in the model class.

Papadimitriou and Lombaert [18] included the effect of spatially correlated prediction errors in an entropybased measurement-system-design methodology. They have shown that the minimum distance between sensors is governed by the spatial correlation length of the prediction errors and thus, by accounting for it, redundancy of information is avoided. In addition, they observed that assumptions of uncorrelated prediction errors in models may lead to sub-optimal measurement systems. Papadopoulou et al. [20] underlined limitations associated with potential redundancy of information using individual-sensor entropy metric. They proposed a methodology that maximizes the joint entropy between sensor locations and includes effects of systematic modeling errors. This study has shown that the joint-entropy design criterion is able to improve model predictions at unmeasured locations compared with individual entropy maximization.

Other studies have focused on sensor locations of high energy content for the record of dynamic properties [13] and parameter grouping techniques [36]. Several researchers have concentrated on global-search optimization algorithms to determine optimal configurations [11, 35, 37, 38]. However, many authors have preferred faster sequential algorithms to global search algorithms $[7,15,18,19]$. Sequential algorithms such as greedy algorithms are part of the family of methods for heuristic optimization. Their search for near optimal solutions often led to good approximations of the optimal measurement-system configuration [18].

Methodologies mentioned above focus on the sensor information accuracy to design measurement systems. As emphasized by Pozzi and Der Kiureghian [27], the value of information should reside in its potential to guide decisions. As a result, measurement systems should be designed for their intended usage. However, in the context of civil structures, few methodologies have quantified the value of information provided by measurement systems, except some authors who evaluated the economic value of the information acquired 
by on-site sensors $[28,40]$.

Recognizing this aspect, Goulet and Smith [7] proposed a methodology where the usefulness of a measurement system is quantified through the metric of expected identifiability. This approach is able to quantify over-instrumentation. Goulet and Smith [7] demonstrated that over-instrumentation appears when the new information provided by additional measurements is exceeded by the amount of uncertainty induced by the inclusion of new measurements. In addition, the methodology includes systematic modeling uncertainties and determines good trade-offs between interpretation goals and available economic resources. However, the approach is limited to maximizing the expected performance of behavior diagnosis.

For model-based data interpretation, the optimal solution is usually related to the way structural-behavior diagnosis and prognosis are evaluated. In addition, most of existing measurement-system-design strategies are based on minimization of the model-parameter uncertainty at the diagnosis stage. However, reducing the uncertainty in remaining-life prognosis of structural components is often more relevant since remaininglife prognosis is able to support decisions related to activities such as repair. In addition, in the context of civil structures, physics-based models are often associated with systematic errors, thus minimizing parameter uncertainty is not judicious. Also, due to the knowledge-intensive nature of the diagnosis and prognosis tasks, such approaches are inadequate for civil structures because their solutions implicitly assume the complete knowledge of errors. Thus, methodologies including engineering heuristics should be favored [24].

This paper proposes a novel strategy for sensor placement that is based on the expected utility of measurement systems. Physics-based-model parameters are identified using error-domain model falsification, a data-interpretation technique that combines engineering knowledge with measurement and models [9]. A population-based prognosis methodology is then used to predict the remaining life of structural components $[22,23]$. Then, this prognosis is used to derive the total repair costs, including monitoring costs, and further the expected utility of measurement systems. A greedy algorithm is used to evaluate optimized solutions to the sensor-placement problem. This approach is able to evaluate whether or not monitoring actions are profitable and when measurement-system performance is reduced by over-instrumentation.

Section 2 presents a background summary of utility theory and a description of system identification through model falsification. Section 3 describes the measurement-system-design methodology combining utility theory and behavior prognosis (Section 3.1). The methodology is illustrated for the case of remainingfatigue-life prognosis of critical connections. The optimization strategy is then presented in Section 3.2. Finally, an application of this methodology to the repair of critical connections of a full-scale case study is presented in Section 4. 


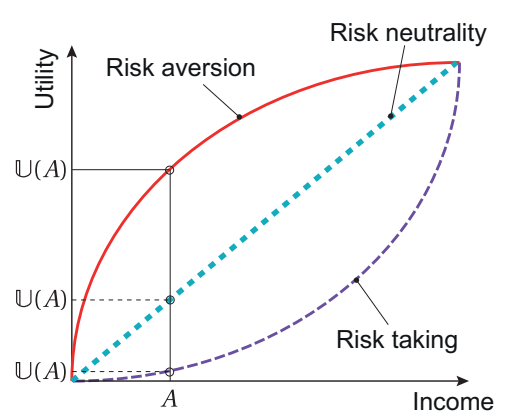

(a)

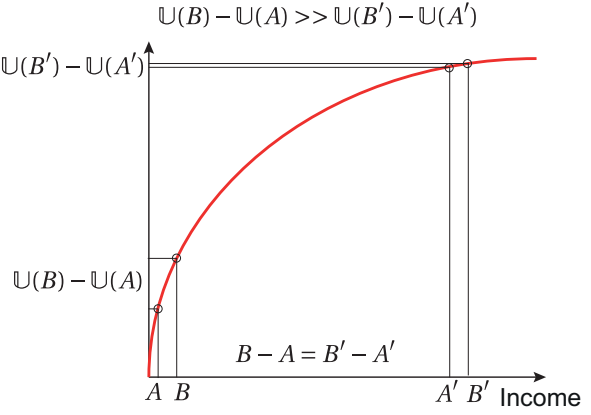

(b)

Figure 1: Schematic comparison of risk-averse, risk-neutral and risk-taking utility functions. (a) Under uncertainty, between a certain but low income $A$ and a higher expected income, a risk-averse person finds more useful the certain income than a risk-neutral and a risk-taking person. (b) Marginal utility depends on the magnitude of income. For example, when $A=0 \$$ and $B=1 \$$, the change in utility $\mathbb{U}(B)-\mathbb{U}(A)$ will be much higher than if $A^{\prime}=100 \$$ and $B^{\prime}=101 \$$ in the case of a risk-averse behavior.

83

\section{Background}

This section presents a background in utility theory and system identification. Subsection 2.1 summarizes decision making through the use of utility functions. Also, a general presentation of system identification and how this task is solved through model falsification are made in Subsection 2.2.

\subsection{Utility theory}

In the field of decision theory, a rational approach for evaluating potential alternative actions when the consequences of choices are not known is the combination of utility theory with probabilities. When making decisions under risk, the possible outcomes are evaluated through their utility and their probability of occurrence [14]. A convenient manner to describe preferences among solutions is the utility function. Utility functions provide a ranking of elements of a choice set. They describe the risk attitude of the decision maker. Three types of risk attitudes are defined: (1) risk averse, (2) risk neutral, and (3) risk taking. A risk-averse person has a preference for an income that is certain over an uncertain income with a larger expected value. For this reason, risk-averse utility functions are monotone and quasi-concave as shown in Figure 1 . When

making decisions under risk, between a certain but low income $A$ and a higher expected income, a risk-averse person finds the certain income to be better than a risk-neutral and a risk-taking person. In addition, if, for two incomes, $A=0 \$$ and $B=1 \$$, the change in utility $\mathbb{U}(B)-\mathbb{U}(A)$ will be much higher than if $A^{\prime}=100 \$$ and $B^{\prime}=101 \$$ in the case of a risk-averse behavior. 
In the management of risky situations, where decisions are made under uncertainty, the expected utility is usually assessed. Expected utility functions are commonly used in economics and finance to model human behavior in relation with money. Utility functions have also been used to assess the engineering performance in construction projects [6], for structural inspection planning [5] and risk assessment of structural failure $[2]$.

\subsection{System identification through model falsification}

Throughout the text, standard variables and indexes are denoted by lower-case letters, e.g. "y, $u, \gamma$ or $\theta$ ". Random variables are denoted by upper-case letters, " $Y, U, \Gamma$ or $\Theta$ ", and realizations of random variables are denoted by " $\tilde{y}, \tilde{u}, \tilde{\gamma}$ or $\tilde{\theta}$ ". Thus, a probability density function (PDF) is described by letter " $f$ " (for example, $f_{U}(u)$ ). Estimations are denoted by a hat symbol, i.e. " $\hat{\mathrm{y}}$ " and average values by an over-line symbol, i.e. " $\bar{\gamma}$ or $\bar{\theta}$ ". Vectors, matrices and sets are represented by bold characters, i.e. "y, $\tilde{\mathbf{y}}$, or $\mathbf{Y} "$. Matrices and vectors are defined between square brackets and sets between braces.

The goal of system identification is to identify values of a vector of parameters $\boldsymbol{\theta}=\left[\theta_{1}, \theta_{2}, \cdots, \theta_{n_{\theta}}\right]^{\top}$ that is used in a model class $g(\cdot)$ based on observations of the structural responses $\hat{\mathbf{y}}$. In the context of civil structures, such parameters are usually stiffnesses of boundary conditions, material Young's moduli and any physics-based parameter that influences the structural response. Since model predictions are associated with modeling uncertainties, parameters $\theta$ are described by random variables $\Theta=\left[\Theta_{1}, \Theta_{2}, \cdots, \Theta_{n_{\theta}}\right]^{\top}$ for predicting the structural responses $\mathbf{Y}=\left[Y_{1}, Y_{2}, \cdots, Y_{n_{\mathrm{y}}}\right]^{\top}$ so that

$$
\mathbf{Y}=g\left(\mathbf{x}_{m}, \boldsymbol{\Gamma}_{m}, \boldsymbol{\Theta}\right)+\mathbf{U}_{g}
$$

where $\mathbf{U}_{g}=\left[U_{g, 1}, U_{g, 2}, \cdots, U_{g, n_{\mathrm{y}}}\right]^{\boldsymbol{\top}}$ represents the modeling uncertainties and where $\mathbf{x}_{m}=[110 \ldots 1]$ is the indicator matrix describing each of the $n_{\mathrm{y}}$ measured degrees of freedom including the number of load configurations under which the response is measured. In this paper, the size of the single-column matrix $\mathbf{x}_{m}$ is the sum of the number of measurement locations and the number of load configurations. A "1" indicates that the measurement (or the load configuration) is used for identification and a "0" indicates that the measurement (or the load configuration) is discarded. The purpose of the methodology presented in this paper is the optimization of the indicator matrix. The subscript " $m$ " relates to variables that are used in the diagnostics process where measured structural responses are compared with model predictions.

In Eq. (1), the model class $g(\cdot)$ includes also parameters $\boldsymbol{\Gamma}_{m}$. These parameters have secondary influence on the structural responses and are taken into account as modeling uncertainties rather than parameters 
to identify. Although they have individually less influence than parameters $\boldsymbol{\theta}$, combined together such uncertainties may have a high influence on the results of system identification. The distinction between primary and secondary parameters may be made by evaluating the relative importance of each parameter on the structural responses as described in $[22,26]$. These parameters are usually sources of uncertainty such as material Poisson's ratios and geometrical variabilities. They are described by the vector $\gamma=\left[\gamma_{1}, \gamma_{2}, \cdots, \gamma_{n_{\gamma}}\right]^{\top}$ and included as random variables $\boldsymbol{\Gamma}=\left[\Gamma_{1}, \Gamma_{2}, \cdots, \Gamma_{n_{\gamma}}\right]^{\top}$.

In addition, structural responses are also defined by observations of the structural responses $\hat{\mathbf{y}}$ such as

$$
\mathbf{Y}=\hat{\mathbf{y}}+\mathbf{U}_{\hat{\mathbf{y}}}
$$

where $\hat{\mathbf{y}}$ are measured values (estimates of $\mathbf{Y}$ ) and $\mathbf{U}_{\hat{\mathbf{y}}}$ are measurement uncertainties. Eq. (1) and Eq. (2) are used to improve our knowledge of model parameters $\boldsymbol{\theta}$.

The model falsification procedure used here was proposed by Goulet and Smith [9]. This procedure involves falsifying all model instances that are instantiations of parameter-value combination of $\boldsymbol{\theta}$ for which the resulting model prediction cannot explain measurement data given modeling and measurement uncertainties $\mathbf{U}$ (from Eq. 1 and 2). It starts with a target reliability of identification $\phi$ and the generation of an initial set of model instances $\boldsymbol{\Omega}$. Based on measurement locations $i=1, \ldots, n_{\mathrm{y}}$, the set of candidate models obtained after falsification is defined by

$$
\boldsymbol{\Omega}^{\prime \prime}=\left\{\boldsymbol{\theta} \in \boldsymbol{\Omega} \mid \forall i: \operatorname{Pr}\left(u_{i, \text { low }} \leq r_{i} \leq u_{i, \mathrm{high}}\right) \leq \phi^{1 / n_{\mathrm{y}}}\right\}
$$

where the lower and upper threshold bounds $\left[u_{i, \text { low }}, u_{i, \text { high }}\right]$ are implicitly defined through the PDF of the combined uncertainties $f_{U_{i}}\left(u_{i}\right)$ of each measurement location $i$ such that

$$
\forall i: \phi^{1 / n_{\mathrm{y}}}=\int_{u_{i, \text { low }}}^{u_{i, \text { high }}} f_{U_{i}}\left(u_{i}\right) \mathrm{d} u_{i}
$$

3 of tior inter intervals including a probability of $\phi^{1 / n_{y}}$.

Formulations of Eq. (3) and Eq. (4) are based on the equalization of Eq. (1) and (2), so that

$$
g\left(\mathbf{x}_{m}, \boldsymbol{\Gamma}_{m}, \boldsymbol{\Theta}\right)+\mathbf{U}_{g}=\hat{\mathbf{y}}+\mathbf{U}_{\hat{\mathbf{y}}} \Longrightarrow g\left(\mathbf{x}_{m}, \boldsymbol{\Gamma}_{m}, \boldsymbol{\Theta}\right)-\hat{\mathbf{y}}=\mathbf{U}_{\hat{\mathbf{y}}}-\mathbf{U}_{g}
$$


and it follows that residuals $r_{i}$ of Eq. (3) are defined so that

$$
\mathbf{r}=\left[r_{1}, r_{2}, \cdots, r_{n_{\mathrm{y}}}\right]^{\boldsymbol{\top}}=g\left(\mathbf{x}_{m}, \bar{\gamma}_{m}, \boldsymbol{\theta}\right)-\hat{\mathbf{y}}
$$

In Eq. (5), parameters $\gamma_{m}$ are uncertain and represent an uncertainty of model predictions. In order to account for these uncertainties, secondary-parameter uncertainties are calculated as the deviation of model predictions to the predictions including the average values $\bar{\gamma}_{m}$ and are combined with the other uncertainties. Consequently, the combined uncertainties $\mathbf{U}$ are defined using Eq. (5) such that

$$
\mathbf{U}=\left[U_{1}, U_{2}, \cdots, U_{n_{\mathrm{y}}}\right]^{\top}=\mathbf{U}_{\hat{\mathbf{y}}}-\left(\mathbf{U}_{g}+g\left(\mathbf{x}_{m}, \boldsymbol{\Gamma}_{m}, \overline{\boldsymbol{\theta}}\right)-g\left(\mathbf{x}_{m}, \bar{\gamma}_{m}, \overline{\boldsymbol{\theta}}\right)\right)
$$

This formulation is valid for all forms of distributions. All model instances that have been falsified are assigned a probability of 0 so that

$$
\operatorname{Pr}\left(\boldsymbol{\Theta}=\theta \notin \boldsymbol{\Omega}^{\prime \prime}\right)=0
$$

and all parameter values not belonging to the falsified set, $\boldsymbol{\theta} \in \boldsymbol{\Omega}^{\prime \prime}$, are labeled as candidate models and are assigned an equal probability

$$
\operatorname{Pr}\left(\boldsymbol{\Theta}=\boldsymbol{\theta} \in \boldsymbol{\Omega}^{\prime \prime}\right)=\frac{1}{\int_{\boldsymbol{\theta} \in \boldsymbol{\Omega}^{\prime \prime}} d \boldsymbol{\theta}}
$$

Consequently, $\Theta^{\prime \prime}$ is defined as the vector of random variables describing the candidate parameter values of $\boldsymbol{\theta}$ given measurement data, $\hat{\mathbf{y}}$. Its PDF is

$$
f_{\boldsymbol{\Theta}^{\prime \prime}}(\boldsymbol{\theta})= \begin{cases}\frac{1}{\int_{\boldsymbol{\theta} \in \boldsymbol{\Omega}^{\prime \prime}} d \boldsymbol{\theta}}, & \text { if } \boldsymbol{\theta} \in \boldsymbol{\Omega}^{\prime \prime} \\ 0, & \text { otherwise }\end{cases}
$$
mor (1)

\section{Methodology}

This methodology combines model falsification and utility theory for optimizing measurement systems in order to maximize the utility of monitoring actions based on remaining-fatigue-life estimations. The 
improved estimate of $\Theta$, denoted $\Theta^{\prime \prime}$, is then used to perform prognosis on unobserved quantities $Z^{\prime \prime}$ defined so that

$$
\left.Z^{\prime \prime}=h\left(g\left(\mathbf{x}_{p}, \boldsymbol{\Gamma}_{p}, \mathbf{\Theta}^{\prime \prime}\right)+U_{g}\right)\right)+U_{h}
$$

where the function $h(\cdot)$ is a post-processing of predicted structural responses, where $U_{h}$ is a random variable describing the post-processing error. For example, this prognosis may be remaining-fatigue-life estimations and prediction of the repair cost of critical connections. The latter behavior prognosis procedure is presented in Section 3.1. The subscript " $p$ " relates to variables that are used in the prognosis process where structural responses are predicted at unobserved degrees of freedom.

Finally, the goal of this methodology is to provide a framework for optimizing measurement systems, $\mathbf{x}_{m}$ so that the expected utility of the unobserved quantities $Z^{\prime \prime}$ is maximized,

$$
\mathbf{x}_{m}^{*}=\underset{\mathbf{x}_{m}}{\arg \max } \mathbb{E}\left[\mathbb{U}\left(Z^{\prime \prime}\right)\right]
$$

The utility function $\mathbb{U}(\cdot)$ and the expected utility maximization framework are described in Section 3.2. Practical application of the methodology proposed here will often lead to problems that are analytically intractable. Section 3.2.1 presents the formulation of a Monte Carlo estimation method for computing the expectation $\mathbb{E}[\cdot]$ presented in Eq. (12) and Section 3.2.2 describes a greedy algorithm based on sequential optimization to approximate the optimal solution.

\subsection{Behavior prognosis}

Structural-behavior prognosis is useful for determining the utility of monitoring actions. In this paper, we propose to illustrate the methodology through remaining-fatigue-life estimations, presented in Section 3.1.1 and evaluating the expected utility of monitoring actions through repair cost considerations in Section 3.1.2.

\subsubsection{Application to bridge remaining-fatigue-life prognosis}

A prognosis of interest may be the remaining-fatigue-life estimation of bridge critical connections as proposed in [23]. For the prediction of remaining fatigue life, a number of cycles under constant stress-range level can be used. This approach, also described in common construction codes $[1,4,31]$, uses a single traffic-axle loading and constant stress amplitudes to determine the remaining number of cycles to failure. A simplified approach, based on Swiss codes [31], is used here because of the low computational resources required to evaluate the remaining fatigue life of connections. 
Constant stress-ranges are determined by calculating the maximum and minimum stresses resulting from the influence line of the moving traffic-axle loading. The stress-range value is defined by $\Delta \sigma=\sigma_{\max }-\sigma_{\min }$, where $\sigma$ is the stress induced in the critical connection by the axle loading. The relation between the stressrange and the number of cycles to failure $n_{f}$ is given by $n_{f}=a \cdot \Delta \sigma^{-b}$, where $a$ is a constant depending on the detail category and $b$ is a measure of the fatigue crack-growth rate. S-N curves describing this relation are available in construction codes and design guides [31,39]. The remaining fatigue life, $\mathbf{R F L}{ }^{\prime \prime}$, that accounts for the candidate parameter values is determined by

$$
\mathbf{R F L}^{\prime \prime}=k \cdot \boldsymbol{\Delta} \boldsymbol{\sigma}^{-b}=k \cdot\left(\left(g\left(\mathbf{x}_{p}^{\max }, \boldsymbol{\Gamma}_{p}, \boldsymbol{\Theta}^{\prime \prime}\right)+\mathbf{U}_{g}^{\max }\right)-\left(g\left(\mathbf{x}_{p}^{\min }, \boldsymbol{\Gamma}_{p}, \boldsymbol{\Theta}^{\prime \prime}\right)+\mathbf{U}_{g}^{\min }\right)\right)^{-b}
$$

where $k=\frac{70}{2 \cdot 10^{6}} \cdot a$ in order to account for the damage equivalence factor that compensates for the simplified traffic-load model [31]. This factor is calibrated to verify the fatigue resistance for $2 \cdot 10^{6}$ load cycles and a service life of 70 years. In addition, in Eq. (13), $\mathbf{x}_{p}^{\max }$ and $\mathbf{x}_{p}^{\min }$ represents the indicator matrix of the maximum and minimum stresses resulting from the influence line. The modeling uncertainties $\mathbf{U}_{g}^{\max }$ and $\mathbf{U}_{g}^{\min }$ are associated with the maximum and minimum stress predictions. They originate from the same sources that are determined during the model-falsification task and are evaluated for the critical locations studied using engineering heuristics.

\subsubsection{Prognosis of repair cost based on fatigue assessment}

In order to determine the expected utility of measurement systems, it is proposed to evaluate the cost of repair in the future of $n_{p}$ critical connections based on improved remaining-fatigue-life estimations, $\mathbf{R F L} \mathbf{L}^{\prime \prime}=$ $\left[R F L_{1}^{\prime \prime}, \ldots, R F L_{n_{p}}^{\prime \prime}\right]^{\top}$, described in Section 3.1.1. In this way, by improving fatigue assessments using an optimal measurement system, it is possible to evaluate before monitoring if the cost of monitoring actions will be worth postponing the repair. However, this approach is subject to the following starting assumptions:

1. repair of $n_{p}$ connections is possible;

2. monitoring data interpretation results in extending fatigue life compared with design-model provisions;

3. the amount of fatigue-life extension can be determined accurately, thus assuming that the model class $g(\mathbf{x}, \boldsymbol{\Gamma}, \boldsymbol{\Theta})$ is right and that uncertainties are correctly estimated;

4. other structural limit states will not become critical during the time of damage accumulation of critical connections.

Let $c_{r}$ be the cost of repairing a single connection. This operation is made at the end of the connection fatigue life assuming repair would be initiated at the end of the theoretical service life. The present value of 
this investment is less than its value in the future assuming positive discount rates. The principle of present value is widely used in finance to determine the value of an expected income that occurs at a future date. Usually, the present value is defined by

$$
p v=\frac{c_{r}}{(1+d)^{t}}
$$

where $t$ is the number of years between the present date and the date where the cost is worth $c_{r}$ and $d$ is the discount rate for a year that is adjusted for inflation.

When improving the remaining-fatigue-life estimation $R F L_{j}^{\prime \prime}$ of a connection $j$ through structural monitoring, the present value of the repair cost decreases as $t$ increases. Depending on the cost of the measurement system, it may or not be financially profitable to improve remaining-fatigue-life predictions. The total cost is also related to the number of connections to be evaluated. The total cost $Z^{\prime \prime}$ of the monitoring that includes the evaluation of $n_{p}$ critical connections that would need to be repaired is

$$
Z^{\prime \prime}=c_{m}+\sum_{j=1}^{n_{p}} \frac{c_{r}}{(1+d)^{R F L_{j}^{\prime \prime}}}
$$

where $c_{m}$ is the cost of monitoring including the cost of load tests, measurement instruments and data interpretation. The right side of this equation represents the function $h(\cdot)$ of Eq. (11) with $U_{h}=0$ in this case. Note that $Z^{\prime \prime}$ is a random variable since it depends on the random variable $R F L_{j}^{\prime \prime}$.

\subsection{Measurement system optimization}

The measurement system optimization is necessary to identify which structural responses to measure, $\mathbf{x}_{m}$, so that the expected utility of $Z^{\prime \prime}$ is maximized. The expected utility is defined by

$$
\mathbb{E}\left[\mathbb{U}\left(Z^{\prime \prime}\right)\right]=\int \mathbb{U}(z) f_{Z^{\prime \prime}}(z) d z
$$

where the utility function $\mathbb{U}(z)$ includes the preference related to the values of $z$. In several practical cases, $\mathbb{U}(z)$ is non-linear and its first derivative has monotonically decreasing values. A common utility function for describing such a situation is the logarithmic function, $\ln z$. However, since the goal of the methodology is the maximization of the expected utility and since $z$ is related to the cost of repair that needs to be minimized, the expression of the utility is given by

$$
\mathbb{U}(z)=\ln (\text { constant }-z)
$$



trapezoidal distribution between 0.25 and 0.75 .

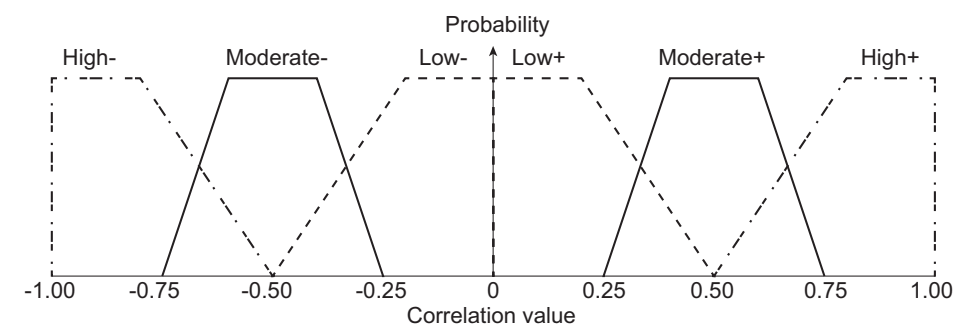

Figure 2: Distribution of correlation values of errors between sensor types and locations within a qualitative reasoning scheme. Users only define qualitatively correlation values between uncertainties by low, moderate, high, negative and positive. Adapted from [8].

With such definition, the utility of a measurement system decreases for increasing total cost $z^{\prime \prime}$. Note that this function is undefined for negative values of $($ constant $-z)$. In order to avoid these values and keep utility calculation unbiased, the constant is chosen to be sufficiently high compared with $z$. Although this modifies the utility value, the relative change in utility values between measurement systems is preserved and this avoids having negative utility values.

\subsubsection{Monte Carlo estimation}

Since at this stage measured values are unknown, Eq. (2) cannot be satisfied based on on-site measurements $\hat{\mathbf{y}}$ and measurement uncertainty $\mathbf{U}_{\hat{y}}$. This pre-posterior analysis requires employing simulated measurements based on the combination of a random selection of $\boldsymbol{\theta}$ and a set of modeling and measurement uncertainties. However, some sources of errors are neither independent nor perfectly correlated between prediction types and position on the structure. The correct correlation values being unknown, the pre-posterior analysis assumes correlation values through qualitative functions.

Figure 2 presents distributions of correlation values between uncertainties that refers to the qualitative reasoning used in [7]. Depending on the source of uncertainty, the correlation value of the errors between sensor types and locations may be low, moderate or high in both signs. The user selects the level of correlation between "Low", "Moderate" and "High", as well as either a positive or a negative correlation. Then, random samples are drawn from the corresponding distribution in Figure 2 that are used when combining uncertainties. For example, for a qualitative "Moderate+" correlation, values are sampled in the

For most practical problems, $\mathbb{E}\left[\mathbb{U}\left(Z^{\prime \prime}\right)\right]$ cannot be estimated analytically for a given measurement configuration $\mathbf{x}_{m}$. A generally applicable alternative is to use Monte Carlo simulations to draw samples of 
simulated measurements from the initial population of model instances $\boldsymbol{\Omega}$, based on Eq. (1) and (2),

$$
\tilde{\mathbf{y}}_{s}=g\left(\mathbf{x}_{m}, \tilde{\gamma}_{m, s}, \tilde{\boldsymbol{\theta}}_{s}\right)+\tilde{\mathbf{u}}_{g, \rho, s}-\tilde{\mathbf{u}}_{\hat{\mathbf{y}}, \rho, s}
$$

where $\tilde{\mathbf{u}}_{g, \rho, s}$ and $\tilde{\mathbf{u}}_{\hat{\mathbf{y}}, \rho, s}$ are samples of measurement and modeling uncertainties (Eq. 7) and the index $\rho$ indicates that uncertainties are correlated through qualitative functions. Simulated measurements lead to identification of a candidate model set $\boldsymbol{\Omega}^{\prime \prime}$. Then, the expected utility can be approximated by

$$
\left.\mathbb{E}\left[\mathbb{U}\left(Z^{\prime \prime}\right)\right] \approx \mathbb{E} \widehat{\left[\mathbb{U}\left(Z^{\prime \prime}\right)\right.}\right]=\frac{1}{n_{s}} \sum_{s=1}^{n_{s}} \mathbb{U}\left(\tilde{z}_{s}^{\prime \prime}\right)
$$

where $\tilde{z}_{s}^{\prime \prime}$ is one of the $n_{s}$ realizations of $Z^{\prime \prime}$ obtained by evaluating the Eq. (11) so that

$$
\tilde{z}_{s}^{\prime \prime}=h\left(g\left(\mathbf{x}_{p}, \tilde{\gamma}_{p, s}, \tilde{\boldsymbol{\theta}}_{s}^{\prime \prime}\right)+\tilde{\mathbf{u}}_{g, s}\right)+\tilde{\mathbf{u}}_{h, s}
$$

using samples simultaneously obtained for $\left\{\tilde{\mathbf{y}}_{s}, \tilde{\gamma}_{m, s}, \tilde{\boldsymbol{\theta}}_{s}, \tilde{\mathbf{u}}_{g, \rho, s}, \tilde{\mathbf{u}}_{\hat{\mathbf{y}}, \rho, s}, \tilde{\gamma}_{p, s}, \tilde{\boldsymbol{\theta}}_{s}^{\prime \prime}, \tilde{\mathbf{u}}_{g, s}, \tilde{\mathbf{u}}_{h, s}\right\}$.

\subsubsection{Measurement-system-optimization strategy}

The space of possible sensor combinations and permutations of measurements grows exponentially with the size of $\mathbf{x}_{m}$. For practical applications, the search space is often discrete and non-convex so that optimization algorithms are necessary to approach the global optimum. In this paper, the measurement-system optimization is performed using a greedy algorithm. A flowchart of the process leading to the maximum expected utility is presented in Figure 3.

This algorithm selects sequentially from an initial sensor configuration $\mathbf{x}_{m}=[11 \ldots 1]$ of $n=n_{\mathrm{y}}$ locations which sensor to remove in order to maximize a single objective, the expected utility. The process of sensor removal continues until a single location is left. In situations where static-load tests are optimized, the process terminates when a single load case and a single sensor remain.

Along with the initial sensor configuration, a set of $n$ sensor configurations is generated with all combinations of $n-1$ sensor locations. For each sensor configuration, the expected utility is computed. The expected utility is calculated using $n_{s}$ draws of simulated measurements referring to the selected sensor configuration. The initial population of model instances is used to randomly select a set of parameters $\tilde{\boldsymbol{\theta}}_{\boldsymbol{s}}$. Then, candidate-model sets are determined by falsifying model instances of the initial population that are not compatible with simulated measurements.

Once the expected value of each sensor configuration containing $n-1$ sensors is computed and stored, 


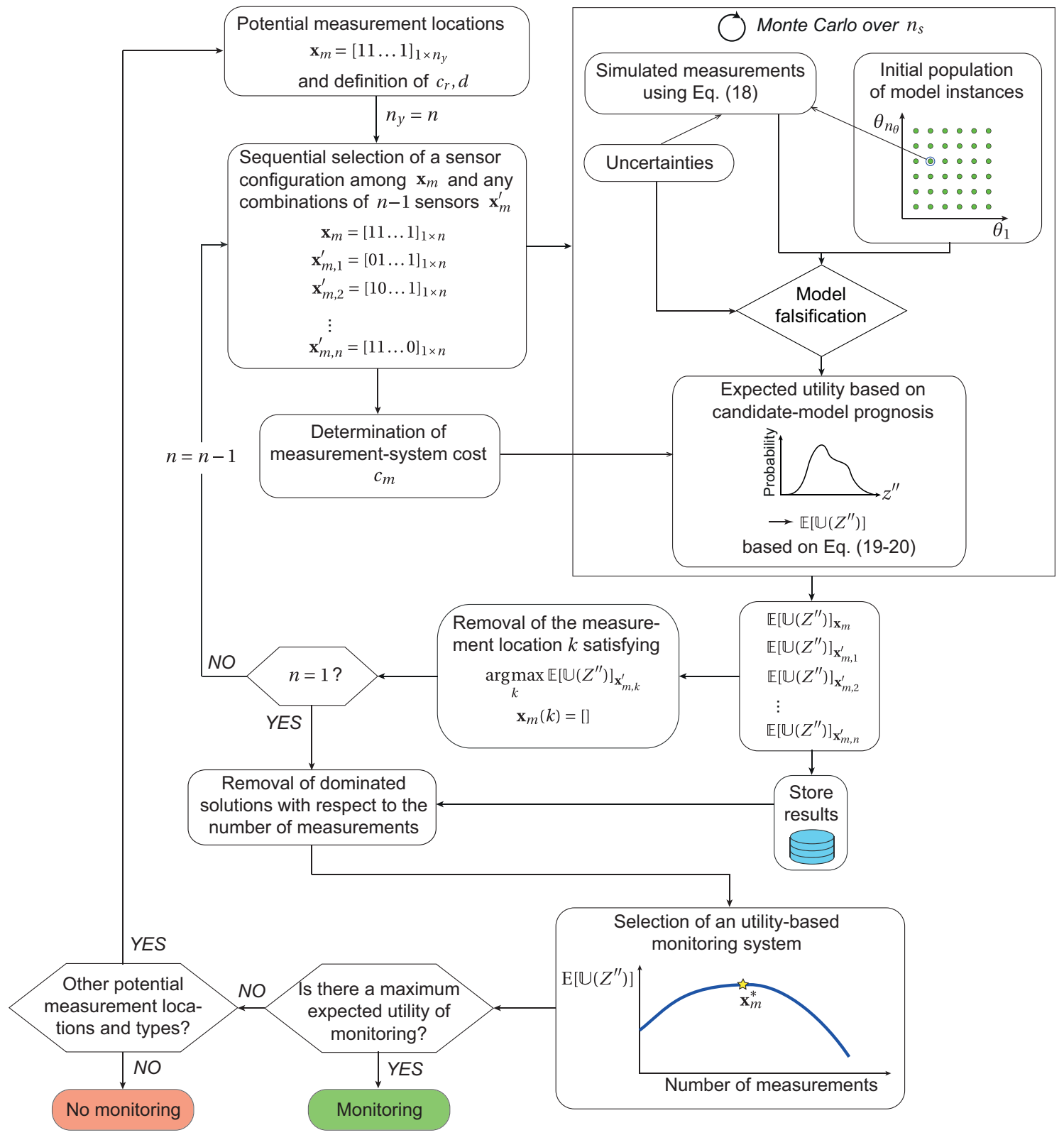

Figure 3: Process flowchart of utility-based measurement-system design using greedy algorithm. 
the algorithm searches for the configurations of $n-1$ sensors that maximize the expected utility. This configuration refers to the removal of sensor location $k$. Since the location $k$ refers to sensor removal among $n$ sensors that lead to the best performance, it is meaningful that the algorithm continues the search for the best expected utility without this sensor location. Although this sensor is permanently removed, the sensor configuration is stored for building the curve of maximal expected-utility solutions at the end of the process.

Thus, the process continues with $n=n-1$ sensors. This process is repeated successively until a single sensor remains. Then, the cost of each sensor configuration that has been computed to determine the expected utility is used to remove solutions that are dominated by a better expected utility. In this way, the optimal expected utility can be paired with its number of measurements including the number of load configurations in order to select the optimal measurement system. This algorithm relies on a backward sequential strategy. This algorithm has a $\mathcal{O}\left(n^{2}\right)$ complexity where $n$ is the number locations times the number of load configurations [7].

When the optimal solution includes a sensor configuration and thus, an expected utility that is greater than the expected utility without a monitoring system, monitoring interventions are profitable. When the optimal solution does not include monitoring actions, it may be possible to search for other potential measurement locations and types and restart the algorithm. If such measurements are not possible, such results justify forgoing monitoring interventions. In such situations, repair costs cannot be reduced by improved evaluation of fatigue reserve capacity, and the prognosis is thus made based on the initial population of model instances $\boldsymbol{\Omega}$.

Also, the curve of the maximum expected utility related to the number of measurements may reveal that measurement systems beyond the optimum decrease the performance of utility and thus, indicate over-instrumentation. Note that the number of measurements includes the number of measured values $n_{y}$ including measurement types and locations as well as load configurations under which the response is measured in case of static-load tests being optimized.

\subsection{The knowledge intensive nature of the methodology}

In this methodology, engineering knowledge and experience are required to estimate PDFs of parameters $\boldsymbol{\Theta}, \boldsymbol{\Gamma}$ and modeling and measurement uncertainties $U_{i, g}$ and $U_{i, \hat{y}}$ as well as qualitative correlations. In complex tasks such as system identification of civil structures, engineering knowledge and heuristics are of utmost importance, particularly in the presence of systematic errors. The advantage of this methodology is that it includes explicit estimates of systematic modeling errors in a simple and robust methodology [9, 24]. 

of the finite-element model and its general overview are displayed in Figure 4.

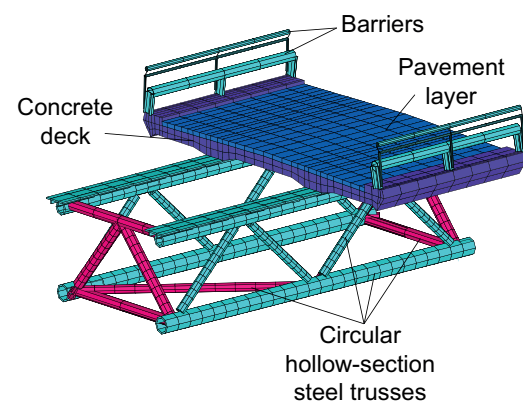

(a)

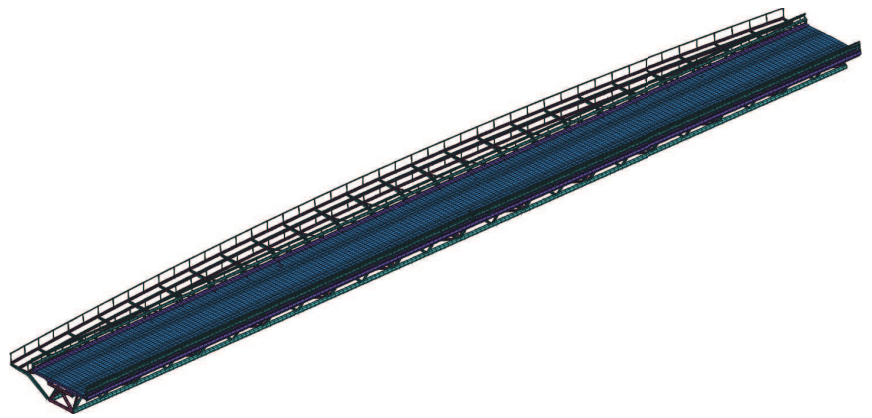

(b)
Figure 4: (a) Aarwangen Bridge model cross-section and (b) general overview. Reprinted with permission from ASCE [23]. and pinned rigid beams such as illustrated in Figure 5. Also, the connection between the concrete deck and

The main assumption that is made is the simplification of the connection behavior using rotational springs

The example that is used to illustrate the measurement-system-design methodology is a composite-steelconcrete bridge located in the city of Aarwangen (Switzerland). The bridge has two spans of $47.8 \mathrm{~m}$ with welded tubular steel trusses connected in a composite manner to the concrete deck that is $8.3 \mathrm{~m}$ wide. A $3 \mathrm{D}$ finite-element model is first built based on the bridge drawings (also described in [22, 23]). The cross-section

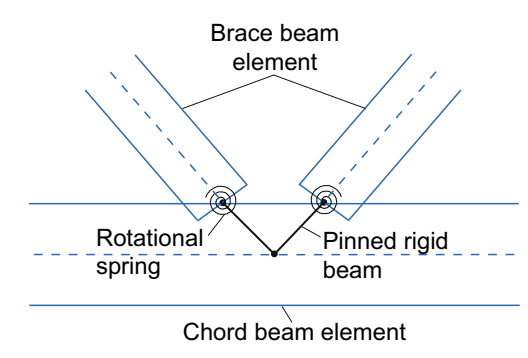

Figure 5: Aarwangen Bridge connection model.

312

\section{Case study}

the steel trusses is assumed to be perfect and the bearing devices are simplified by perfect expansion and fixed supports since they were in good condition.

Several characteristics of the structure are unknown and have potentially a significant influence on the structural response: the rotational stiffness of the truss connections (modeled by springs) and the Young's moduli of concrete and pavement. In addition, an in-situ inspection has highlighted a potential significant 
effect of the longitudinal stiffness of the pavement covering expansion joints. The model class $g(\cdot)$ includes thus these unknown parameters $\boldsymbol{\theta}$. As illustrated in Figure 6, the rotational stiffness of the truss connections is represented by rotational springs connected between the diagonal members and either the upper or lower chord.

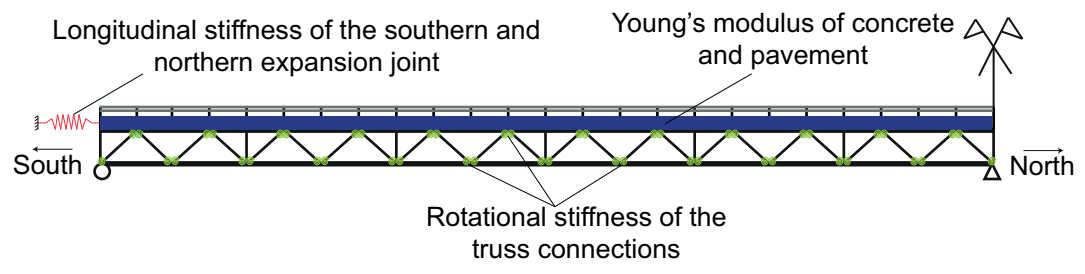

Figure 6: Aarwangen Bridge model parameters. Adapted from [22].

Expansion joints are modeled using springs parallel to the bridge longitudinal axis. Possible parameter values for spring stiffnesses are sought for both the southern and northern abutments. These structural properties are to be identified using behavior measurements. Other uncertainties, such as steel Young's modulus, concrete Poisson's ratio, truck weight and member dimensions, have a secondary influence on the structural behavior and are modeled by parameters $\gamma$.

The goal of this study is the determination of the best measurement system (load-test and sensor configuration) among potential load cases, sensors and sensor locations through maximizing the expected utility of monitoring. The purpose of monitoring actions is the maximization of the expected utility based on repair cost of the four critical connections displayed in Figure 7.

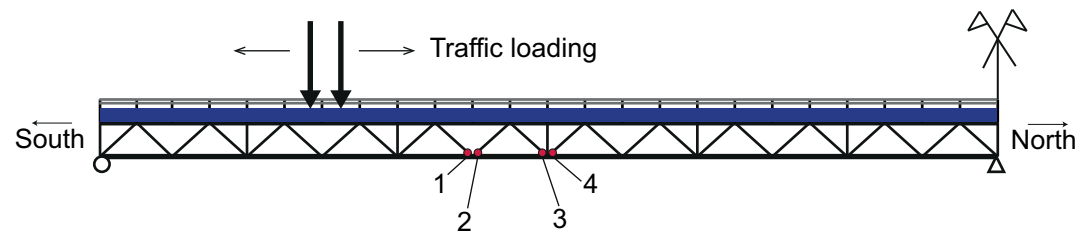

Figure 7: Aarwangen Bridge critical connections and code traffic loading. Adapted from [23].

Initial parameters values $\boldsymbol{\theta}=\left[\theta_{1}, \theta_{2}, \ldots, \theta_{5}\right]$ and their initial range of values estimated based on engineering experience are presented in Table 1 . PDFs of secondary parameter random variables $\Gamma$ are displayed in Table 2. These PDFs are estimated using engineering knowledge [23]. Note that the variability of profile thickness $\gamma_{m, 4}$ and diameter $\gamma_{m, 6}$ of critical connections under study are not taken into account during prognosis of remaining fatigue life since such uncertainties are already included in the S-N curve elaboration. 
Table 1: Initial-model parameter ranges for the Aarwangen Bridge

\begin{tabular}{lcccc}
\hline Parameter $\theta$ & Units & PDF & Min & Max \\
\hline$\Theta_{1}:$ Rotational stiffness of truss connections & $\mathrm{MNm} / \mathrm{rad}$ & Uniform & $10^{-1}$ & $10^{3}$ \\
$\Theta_{2}:$ Stiffness of southern expansion joint & $\mathrm{MN} / \mathrm{m}$ & Uniform & $10^{-1}$ & $10^{4}$ \\
$\Theta_{3}$ : Stiffness of northern expansion joint & $\mathrm{MN} / \mathrm{m}$ & Uniform & $10^{-1}$ & $10^{4}$ \\
$\Theta_{4}$ : Young's modulus of concrete & $\mathrm{GPa}$ & Uniform & 20 & 50 \\
$\Theta_{5}:$ Young's modulus of pavement & $\mathrm{GPa}$ & Uniform & 2 & 20 \\
\hline
\end{tabular}

Table 2: Aarwangen Bridge secondary parameter random variables $\gamma$ and their PDF as inputed in the Monte Carlo simulation.

\begin{tabular}{llcccc}
\hline Parameters $\gamma_{m}, \gamma_{p}$ & Description & Unit & PDF & Mean/Min & SD/Max \\
\hline$\Gamma_{m, 1}, \Gamma_{p, 1}$ & Young's modulus of steel & GPa & Gaussian & 207 & 2 \\
$\Gamma_{m, 2}, \Gamma_{p, 2}$ & $\Delta v$ Poisson's ratio of concrete & - & Gaussian & 0.19 & 0.025 \\
$\Gamma_{m, 3}, \Gamma_{p, 3}$ & $\Delta t$ pavement thickness & $\%$ & Gaussian & 0 & 2.5 \\
$\Gamma_{m, 4}$ & $\Delta t_{1}$ steel profile thickness & $\%$ & Uniform & -10 & 10 \\
$\Gamma_{m, 5}, \Gamma_{p, 4}$ & $\Delta t_{2}$ steel profile thickness & $\%$ & Uniform & -12.5 & 12.5 \\
$\Gamma_{m, 6}$ & $\Delta D_{1}$ steel profile diameter & $\%$ & Uniform & -1 & 1 \\
$\Gamma_{m, 7}, \Gamma_{p, 5}$ & $\Delta D_{2}$ steel profile diameter & $\%$ & Uniform & -1 & 1 \\
$\Gamma_{m, 8}$ & $\Delta W$ truck-axle weight & $\mathrm{kN}$ & Uniform & -1 & 1 \\
\hline
\end{tabular}

In order to test the measurement-system-design methodology described in Section 3, four potential load configurations are proposed in Figure 8. Each load case involves four trucks positioned either symmetrically or asymmetrically with respect to bridge traffic lanes.

Potential sensor locations and types are shown in Figure 9. Overall 30 sensor types and locations are possible, including four displacement sensors, nine inclinometers and 17 strain gages. Along with the four load-test configurations, 120 measurements are possible. Displacement gages are positioned to measure the displacement of the southern and northern expansion joint. Inclinometers are located on the bottom chord of east-side truss. Strain gages are located in the middle of the truss braces on the east and west side since it has been noted by previous studies that parameter values were more sensitive at those locations [23].

The cost of a connection repair is assumed to be $c_{r}=\$ 15000$ and the discount rate is taken to be $d=1 \%$ which represents a good approximation of Swiss interest rates including the effect of inflation. It is assumed that displacement sensors, inclinometers, strain gages cost respectively $\$ 200, \$ 600$ and $\$ 200$. Also, each truck is expected to have a fix cost $\$ 400$ and then $\$ 200$ per hour of use. Each load case lasts two hours. These values are based on [7].

The initial population of models is generated with 3125 instances based on the uniform sampling of the initial ranges displayed in Table 1. This number of models is obtained by dividing the five initial ranges into 


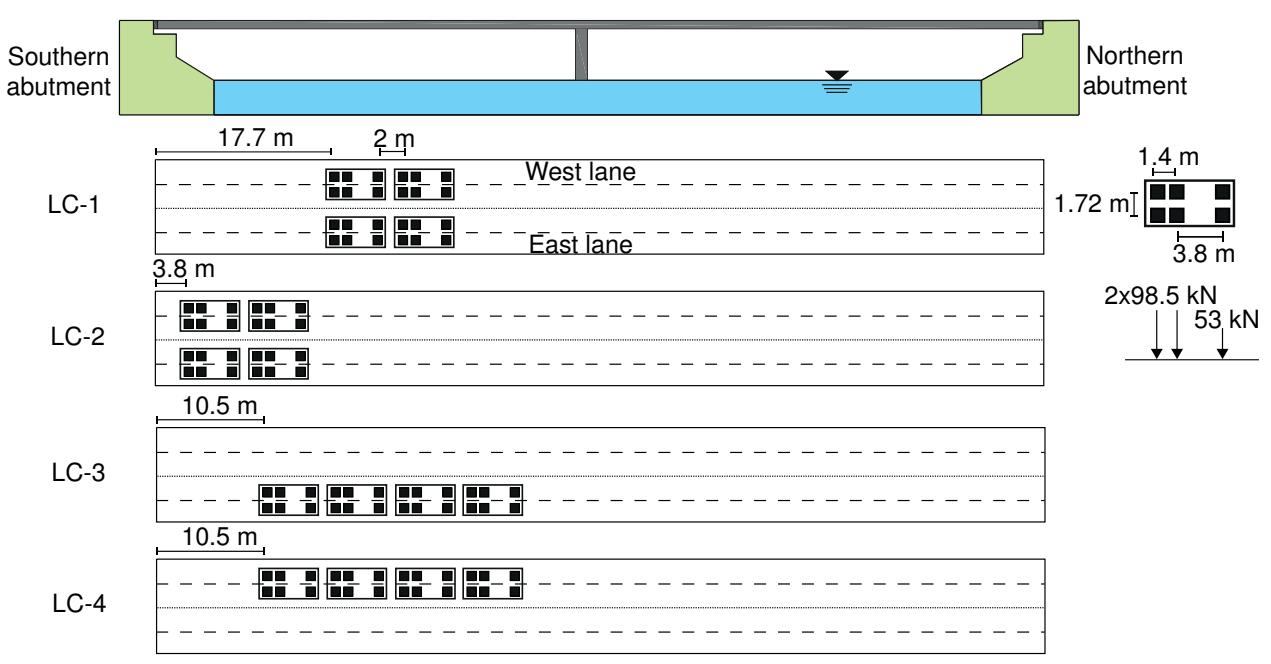

Figure 8: Potential load-test configurations for measurement-system design.

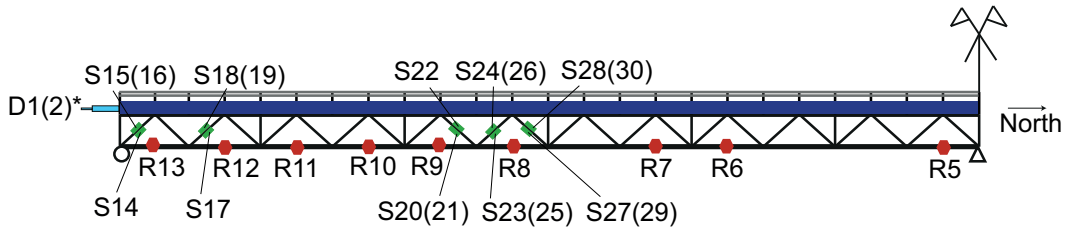

East truss elevation (sensor numbers in parenthesis are located on west truss)

$\Longrightarrow$ Displacement gauge D.. (*also at northern abutment D3(4))

- Inclinometer R..

- Strain gauge S..

Figure 9: Potential sensor locations and types for measurement-system design. 
the qualitative estimate of correlations based on Figure 2.

Table 3: Other sources of uncertainty involved in the measurement-system design for each sensor type and qualitative correlation values assigned between sensor types for the Aarwangen Bridge.

\begin{tabular}{lccccccc}
\hline & \multicolumn{2}{c}{ Displacement $[\mathrm{mm}]$} & Rotation $[\mu \mathrm{rad}]$ & \multicolumn{2}{c}{ Strain $[\mu \epsilon]$} & Correlation \\
\cline { 2 - 8 } Uncertainty sources $U_{i, g}$ and $U_{i, \hat{y}}$ & Min & Max & Min & Max & Min & Max & qualitative \\
\hline Model simplifications [\%] & 0 & 2 & 0 & 2 & 0 & 5 & High+ \\
Mesh refinement [\%] & -2 & 0 & -2 & 0 & -2 & 0 & High+ + \\
Additional uncertainty [\%] & -1 & 1 & -1 & 1 & -1 & 1 & Moderate+ \\
\cline { 2 - 8 } & Mean & SD & Mean & SD & Mean & SD & \\
Sensor resolution [absolute unit] & 0 & 0.1 & 0 & 2 & 0 & 1 & Low+ \\
Cable losses [\%] & 0 & 0 & 0 & 0 & 0 & 1 & Low + \\
Repeatability [\%] & 0 & 1.5 & 0 & 1.5 & 0 & 1.5 & High+ \\
Truck position [\%] & 0 & 1.5 & 0 & 1.5 & 0 & 1.5 & Moderate+ \\
\hline
\end{tabular}

Table 3 presents uncertainties and correlations that are estimated using engineering judgment and field observations and included in the methodology. This way of estimating sources of errors allows for a form of uncertainty distribution that is physically appropriate for each source [25]. In addition, it enables the modification of a distribution when new information becomes available within the iterative process of system identification [26].

Model simplifications, mesh refinement and measurement repeatability are sources of uncertainty that may have high interaction between measurement types and locations so that a high correlation is assigned. Conversely, sensor resolution and cable losses induce uncertainties that are almost independent and therefore, a low correlation is assigned. The other sources may have a moderate degree of correlation. Note that all correlation values are assumed positive.

The lower part of Table 3 summarizes also the uncertainty distributions for measurement errors. These uncertainties are estimated using engineering heuristics and field conditions. They are combined with modeling uncertainties using Eq. (7) in order to determine the threshold bounds in the falsification process for each loading scenarios and sensor position $\mathbf{x}_{m}$. During the combination, uncertainties in percentage are multiplied with the predictions of the model having the mean parameter values in order to estimate the uncertainty in absolute units for any loading level, scenarios and sensor type. 


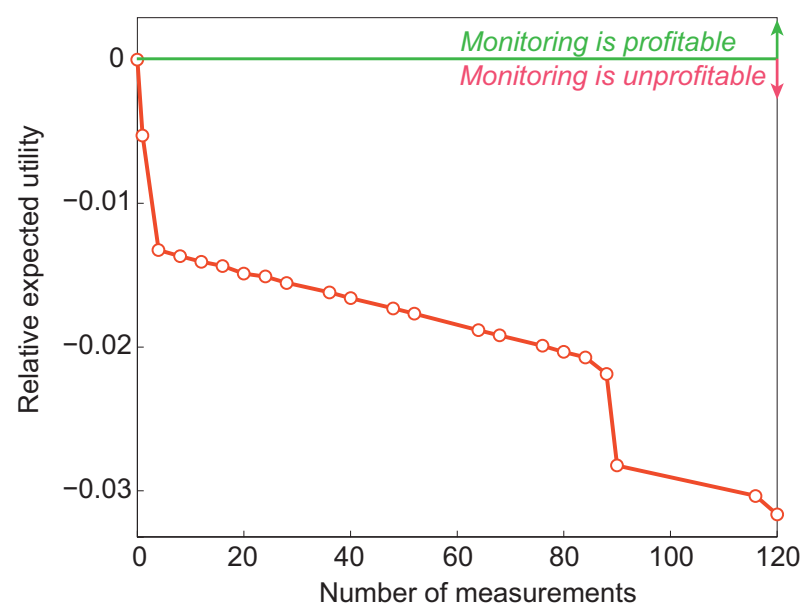

Figure 10: Expected utility in relation with the number of measurements for the Aarwangen Bridge. The almost linear behavior of the solutions are insensitive to change in remaining-fatigue-life predictions between sensor configuration and thus, are dominated by the load test costs. The monotonically decreasing relative expected utility with respect to costs indicate that any monitoring intervention is unprofitable.

The greedy algorithm is launched with the initial sets of all load-case and sensor configurations. For each set of simulated measurements, model instances are falsified using Eq. (3) with $\phi=0.95$. Depending on the number of measurements, coverage regions are adjusted according to $\phi^{1 / n_{\mathrm{y}}}$ (Eq. 4), such that more measurements lead to wider coverage regions. The remaining fatigue life is then computed using the obtained candidate-model set using the constant nominal stress-range approach (Section 3.1.1) and using identical accommodation of modeling uncertainties. The process then continues with expected-utility maximization and sensor removal as explained in Section 3.2.2 until a single load case and sensor location are left.

\subsection{Aarwangen Bridge sensor placement}

Figure 10 presents the results of the measurement-system design for the four critical connections of the Aarwangen Bridge. This figure shows the overall number of measurements including the number of load configurations used for data interpretation in relation with the maximum expected utility. Note that the expected utility is represented relative to the value computed without monitoring actions in order to have zero utility when no measurement is taken. It is observed that each measurement system leads to an expected utility that is below the utility without monitoring. This means that any measurement system is unprofitable since the optimum expected utility is obtained when no measurement is taken.

The linear trend of the curve reveals a domination of the monitoring cost $c_{m}$ over the repair costs. The reserve capacities of these connections are around 500 years, meaning that the actualization of the repair cost 
leads to very low values. This is due to the division by $(1+0.01)^{500}$ in Eq. (15), which leads to an actualized repair cost of approximately US\$580 when summed for the four connections. This is understandable that an outcome in 500 years has a very low present value. Thus, since monitoring costs vary between US\$3400 and 17600 , the effect of actualized repair costs is low such that monitoring interventions would be unprofitable for any measurement system. In this curve, the monitoring $\operatorname{cost} c_{m}$ is mainly related with $\ln \left(\right.$ constant $\left.-c_{m}\right)$ leading to logarithmic-curve segments that seem linear.

These results demonstrate that monitoring interventions are not always necessary and profitable. This methodology is thus able to detect monitoring of infrastructure that is unjustified according to utility as defined in this paper.

\subsection{Modified Aarwangen Bridge sensor placement}

In order to illustrate the potential of this approach when monitoring actions are profitable, an example is simulated from the Aarwangen Bridge model predictions. The amplitude of stress-ranges are multiplied by three for all initial model instances. The remaining-fatigue-life predictions are thus reduced. This case has higher actualization values of the repair costs and is more representative of situations common to aging infrastructure. Also, the number of critical connections to evaluate is increased to 40 by replication of available stress-ranges.

Equivalent assumptions are made regarding $c_{r}, d$ and the costs of trucks and sensors. Figure 11 presents the results of the measurement-system design for this illustrative example.

It is shown that monitoring may be useful and profitable. Indeed, for a number of measurements lesser than 30, the expected utility is larger than the utility without monitoring such that most measurement systems are in the profitable region. For a number of measurements greater than 30, monitoring interventions and data interpretation are unprofitable. In addition, the methodology is able to identify an optimum measurement system for six measurements. The measurement system that leads to the best expected utility is obtained using two displacement sensors (D1 and D2) and one strain gage (S24) under LC-1 and LC-2.

In addition, it is shown that, beyond this optimum, adding more measurements will lead to overinstrumentation as represented by the performance decrease. Indeed, for higher investments in monitoring, the expected utility of such measurement systems becomes equivalent to the situation of lower monitoring investment. The optimum means that a present investment of US\$5400 for a monitoring system of three sensors and two load cases will lead to profitable repair in the future since the money saved by avoiding an early repair can be invested with return of interest. Table 4 presents the optimal measurement configurations obtained in Figure 11. 


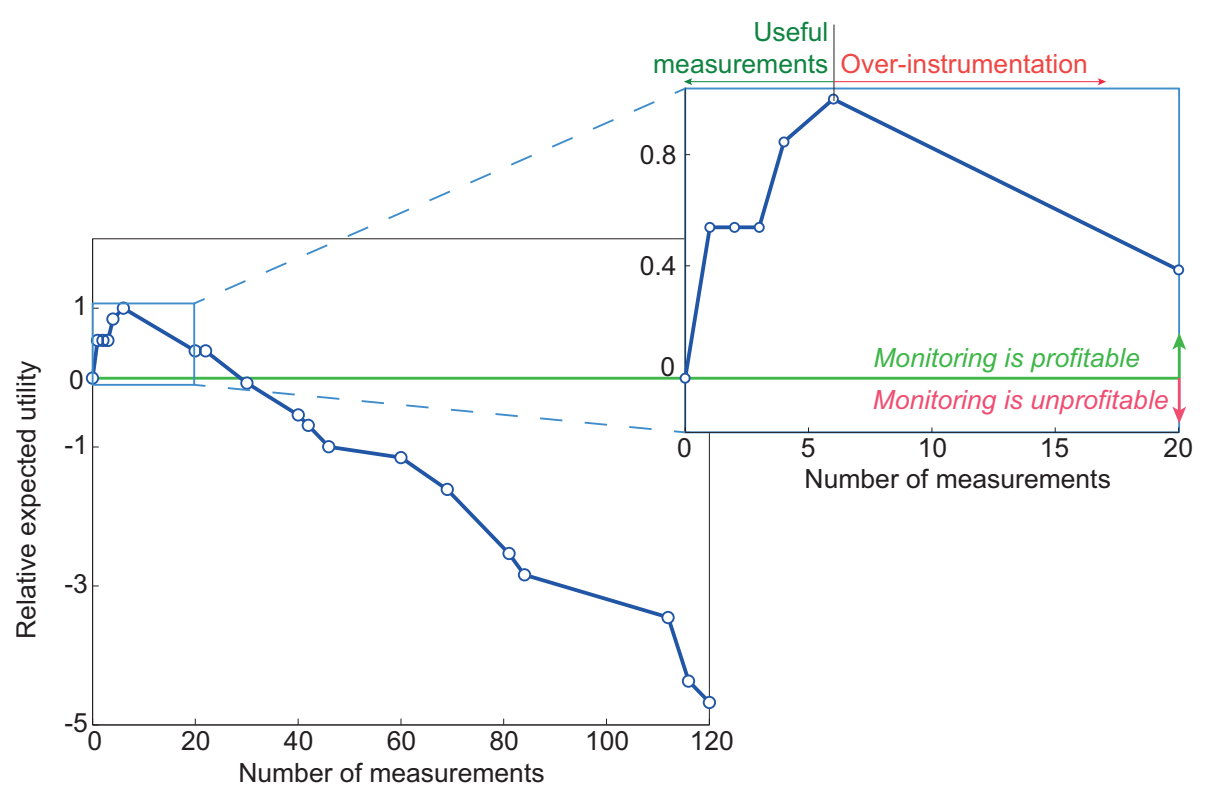

Figure 11: Expected utility in relation with load test costs for an illustrative example based on the Aarwangen Bridge. An optimum measurement system is found for six measurements. For a lesser number of measurements than this optimum, measurements are useful and profitable. Adding more measurements would reduce the efficiency of monitoring interventions and data interpretation even if the monitoring is profitable. For more than 30 measurements, monitoring becomes unprofitable. 


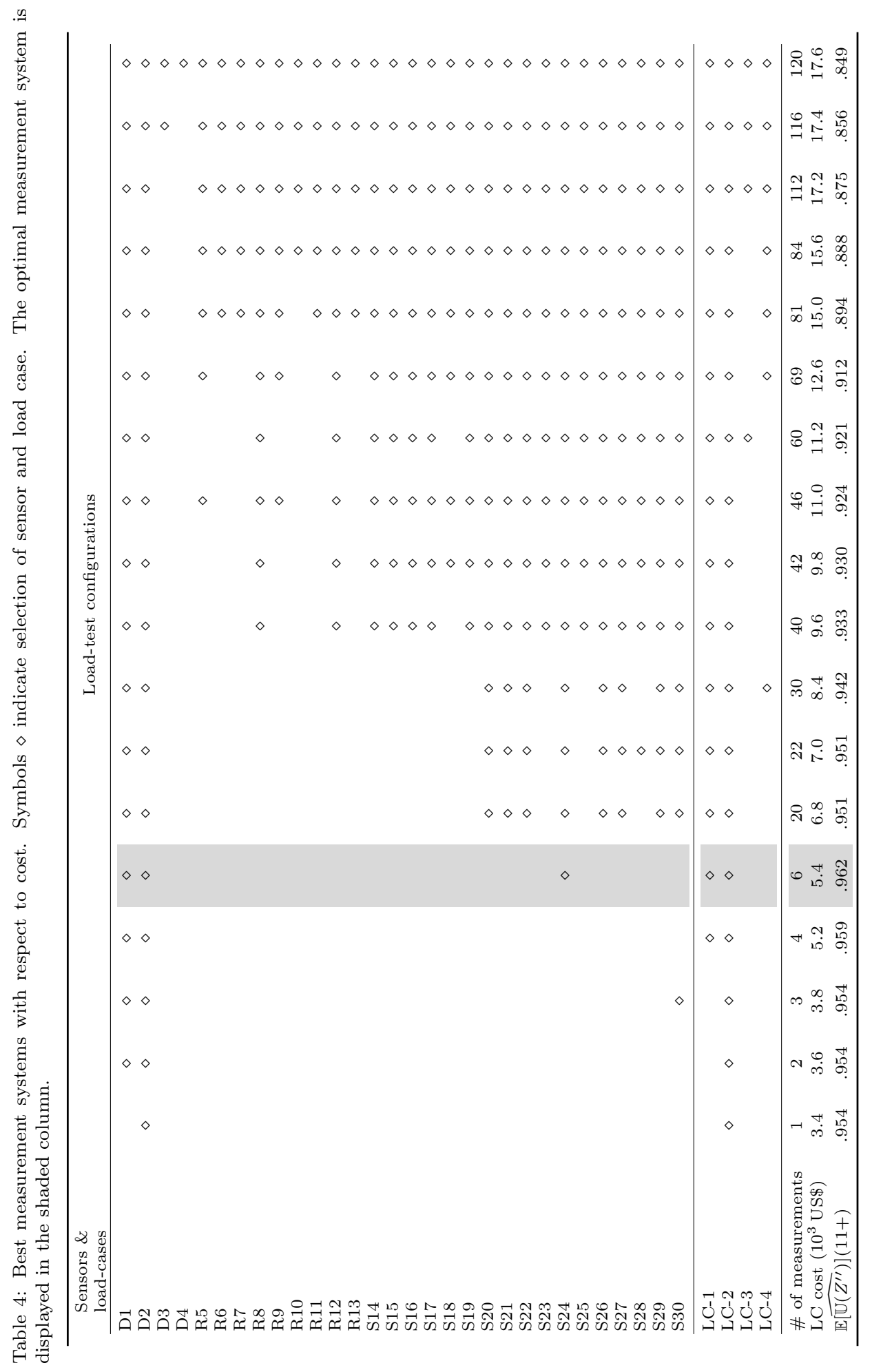




\section{Discussion}

The utility-based measurement-system-design strategy described in this paper involves the assumption of a constant discount rate during the period of fatigue damage accumulation that may theoretically last for centuries. Although the assumption of having the same discount rate 100 years including inflation rate from now is weak and may bias the expected utility values, the optimal measurement system is insensitive to the choice of the discount rate even for varying values throughout time. This argument has been verified with the example of the Aarwangen Bridge for discount rates of $2 \%$ and $5 \%$ leading to the same optimal measurement system as the results with $d=1 \%$. The difference resides only in the absolute values of expected utility and in a variation in the extent of the profitable monitoring region.

In addition, the choice of a logarithmic function as the utility function may be also arbitrary. However, the logarithmic function, apart from the advantage of describing a risk-averse behavior of the decision maker, allows for assigning a larger expected utility to total cost $Z^{\prime \prime}$ that have less dispersion for equivalent expected value such that a certain total cost has a higher utility than an uncertain total cost.

Compared with other approaches presented in Section 1, this methodology provides a direct evaluation of the utility of measurement systems. Although entropy-based techniques are appropriate for the determination of the most informative measurement systems, the estimation of the expected utility based on the cost of improving remaining-fatigue-life predictions is efficient to support engineering decision-making in a more comprehensive way. This methodology may be adapted to other types of time-dependent prognoses subject to similar starting conditions listed in Section 3.1.2. For example, the calculation of the repair costs could be adapted to the prognosis type.

This methodology requires engineering knowledge for building the model class, for defining initial-model parameter ranges, for identifying sources of errors, for estimating modeling and measurement errors during identification and prediction and as well for evaluating repair costs. The engineering knowledge, which is indirectly and partially introduced in common approaches, is explicitly represented in this methodology and this allows ease of modification when new information is acquired.

Since uncertainty values are often determined heuristically, it is of interest to study the sensitivity of the results to variations in uncertainty ranges and correlations. A sensitivity analysis of the results revealed that the method is robust to under and over-estimation of model simplification uncertainties through varying uncertainty ranges in four steps from 0 to $100 \%$. The same optimal solution was identified at each step. However, sensitivities with respect to correlations and systematic biases were not studied and this is the subject of future research. More detail can be found in [21]. 


\section{Conclusions}

The maximization of expected utility allows evaluation of expected improvements in critical limit states such as fatigue reserve capacity in terms of future infrastructure repair costs. Conclusions are as follows:

- The utility-based measurement-system-design methodology proposed is able to quantify the optimal expected utility of monitoring actions using remaining-fatigue-life estimations and cost considerations.

- This strategy successfully supports evaluations of whether or not monitoring actions are profitable and when measurement-system performance becomes reduced by over-instrumentation.

- Through determining the expected utility of monitoring actions at the prognosis stage, this methodology provides direct guidance related to decision making for structural management while current approaches that are limited to optimization at the diagnosis-stage may not provide such support.

- The use of model falsification for system identification allows for explicit inclusion of engineering knowledge and heuristics so that new information can be integrated effectively. This is particularly helpful when measurement systems are re-evaluated over several years of measurement diagnoses and behavior prediction.

\section{Acknowledgements}

This work was funded by the Swiss National Science Foundation under Contract no. 200020-155972.

\section{References}

[1] AASHTO, 2007. LRFD Bridge Design Specifications. 4th Edition, American Association of State Highway and Transportation Officials, Washington, DC.

[2] Brito, A., de Almeida, A., 2009. Multi-attribute risk assessment for risk ranking of natural gas pipelines. Reliability Engineering \& System Safety 94 (2), 187-198.

[3] Brownjohn, J., 2007. Structural health monitoring of civil infrastructure. Philosophical Transactions of the Royal Society A: Mathematical, Physical and Engineering Sciences 365 (1851), 589-622.

[4] EN1993-1-9, 2005. Eurocode 3 - Design of steel structrures - Part 1-9: Fatigue. European committee for standardization.

[5] Ferreira, R., de Almeida, A., Cavalcante, C., 2009. A multi-criteria decision model to determine inspection intervals of condition monitoring based on delay time analysis. Reliability Engineering \& System Safety 94 (5), 905-912. 
[6] Georgy, M., Chang, L.-M., Zhang, L., 2005. Utility-function model for engineering performance assessment. Journal of Civil Engineering and Management 131 (5), 558-568.

[7] Goulet, J.-A., Smith, I. F. C., 2013. Performance-driven measurement system design for structural identification. Journal of Computing in Civil Engineering 27 (4), 427-436.

[8] Goulet, J.-A., Smith, I. F. C., 2013. Predicting the usefulness of monitoring for identifying the behavior of structures. Journal of Structural Engineering 139 (10), 1716-1727.

[9] Goulet, J.-A., Smith, I. F. C., 2013. Structural identification with systematic errors and unknown uncertainty dependencies. Computers \& Structures 128, 251-258.

[10] Heredia-Zavoni, E., Montes-Iturrizaga, R., Esteva, L., 1999. Optimal instrumentation of structures on flexible base for system identification. Earthquake engineering \& structural dynamics 28 (12), 1471-1482.

[11] Kang, F., Li, J.-J., Xu, Q., 2008. Virus coevolution partheno-genetic algorithms for optimal sensor placement. Advanced Engineering Informatics 22 (3), 362-370.

[12] Kripakaran, P., Smith, I. F. C., 2009. Configuring and enhancing measurement systems for damage identification. Advanced Engineering Informatics 23 (4), 424-432.

[13] Meo, M., Zumpano, G., 2005. On the optimal sensor placement techniques for a bridge structure. Engineering Structures 27 (10), 1488-1497.

[14] North, D., 1968. A tutorial introduction to decision theory. IEEE Transactions on Systems Science and Cybernetics 4 (3), 200-210.

[15] Papadimitriou, C., 2004. Optimal sensor placement methodology for parametric identification of structural systems. Journal of Sound and Vibration 278 (4), 923-947.

[16] Papadimitriou, C., 2005. Pareto optimal sensor locations for structural identification. Computer Methods in Applied Mechanics and Engineering 194 (12), 1655-1673.

[17] Papadimitriou, C., Beck, J., Au, S.-K., 2000. Entropy-based optimal sensor location for structural model updating. Journal of Vibration and Control 6 (5), 781-800.

[18] Papadimitriou, C., Lombaert, G., 2012. The effect of prediction error correlation on optimal sensor placement in structural dynamics. Mechanical Systems and Signal Processing 28, 105-127.

[19] Papadopoulou, M., Raphael, B., Smith, I. F. C., Chandra, S., 2015. Optimal sensor placement for time-dependent systems: Application to wind studies around buildings. Journal of Computing in Civil Engineering in press, 04015024.

[20] Papadopoulou, M., Raphael, B., Smith, I. F. C., Sekhar, C., 2014. Hierarchical sensor placement using joint entropy and the effect of modeling error. Entropy 16 (9), 5078-5101.

[21] Pasquier, R., 2015. Performance assessment and prognosis for civil infrastructure based on model falsification reasoning. thesis $\mathrm{n}^{\circ} 6756$. Ph.D. thesis, Swiss Federal Institute of Technology (EPFL). 
[22] Pasquier, R., D’Angelo, L., Goulet, J.-A., Acevedo, C., Nussbaumer, A., Smith, I. F. C., 2016. Measurement, data interpretation, and uncertainty propagation for fatigue assessments of structures. Journal of Bridge Engineering, 04015087.

[23] Pasquier, R., Goulet, J.-A., Acevedo, C., Smith, I. F. C., 2014. Improving fatigue evaluations of structures using in-service behavior measurement data. Journal of Bridge Engineering 19 (11), 04014045.

[24] Pasquier, R., Smith, I. F. C., 2015. Robust system identification and model predictions in the presence of systematic uncertainty. Advanced Engineering Informatics 29 (4), 1096-1109.

[25] Pasquier, R., Smith, I. F. C., 2015. Sources and forms of modelling uncertainties for structural identification. In: Proceedings of 7 th International Conference on Structural Health Monitoring of Intelligent Infrastructure (SHMII), Torino, IT.

[26] Pasquier, R., Smith, I. F. C., 2016. Iterative structural identification framework for evaluation of existing structures. Engineering Structures 106, 179-194.

[27] Pozzi, M., Der Kiureghian, A., 2011. Assessing the value of information for long-term structural health monitoring. In: SPIE Smart Structures and Materials+ Nondestructive Evaluation and Health Monitoring. International Society for Optics and Photonics, pp. 79842W-79842W.

[28] Pozzi, M., Zonta, D., Wang, W., Chen, G., 2010. A framework for evaluating the impact of structural health monitoring on bridge management. In: Proc. 5th Int. Conf. Bridge Maintenance, Safety Manage. p. 161.

[29] Qureshi, Z., Ng, T., Goodwin, G., 1980. Optimum experimental design for identification of distributed parameter systems. International Journal of Control 31 (1), 21-29.

[30] Robert-Nicoud, Y., Raphael, B., Smith, I. F. C., 2005. Configuration of measurement systems using Shannon's entropy function. Computers \& structures 83 (8), 599-612.

[31] SIA263 Code, 2003. Norme SIA 263 : Steel structures. SIA Zurich.

[32] Šidák, Z., 1967. Rectangular confidence regions for the means of multivariate normal distributions. Journal of the American Statistical Association 62 (318), 626-633.

[33] Smith, I. F. C., 2016. Studies of sensor-data interpretation for asset management of the built environment. Frontiers in Built Environment 2, 8 .

[34] Stephan, C., 2012. Sensor placement for modal identification. Mechanical Systems and Signal Processing 27, 461-470.

[35] Worden, K., Burrows, A., 2001. Optimal sensor placement for fault detection. Engineering Structures 23 (8), 885-901.

[36] Yeo, I., Shin, S., Lee, H., Chang, S.-P., 2000. Statistical damage assessment of framed structures from static responses. Journal of Engineering Mechanics 126 (4), 414-421.

[37] Yi, T.-H., Li, H.-N., Gu, M., 2011. Optimal sensor placement for structural health monitoring based on multiple optimization strategies. The Structural Design of Tall and Special Buildings 20 (7), 881-900. 
[38] Yi, T.-H., Li, H.-N., Zhang, X.-D., 2015. Health monitoring sensor placement optimization for Canton Tower using immune monkey algorithm. Structural Control and Health Monitoring 22 (1), 123-138.

[39] Zhao, X.-L., Herion, S., Packer, J., Puthli, R., Sedlacek, G., Wardenier, J., Weynand, K., van Wingerde, A., Yeomans, N., 2002. Design guide for circular and rectangular hollow section welded joints under fatigue loading. TÜV-Verlag GmbH.

[40] Zonta, D., Glisic, B., Adriaenssens, S., 2014. Value of information: impact of monitoring on decision-making. Structural Control and Health Monitoring 21 (7), 1043-1056. 